\title{
El rescate de los ingredientes patrimoniales colombianos por medio del turismo gastronómico
}

\section{The rescue of Colombian patrimonial ingredients through gastronomic tourism}

MARÍA ALEJANDRA BALLESTEROS MEJÍA

(D) https://orcid.org/0000-0002-7167-2934

Universidad Agustiniana (Colombia)

maria.ballesterosm@uniagustiniana.edu.co

Artículo de investigación

Recepción: 30 de septiembre de 2020

Aceptación: 09 de febrero de 2021

Cómo citar este artículo

Ballesteros Mejía, M. (2020). El rescate de los ingredientes

patrimoniales colombianos por medio del turismo gastronómico. Sosquua 2(2), pp. 23-32. Recuperado a partir de: http://cipres.sanmateo.edu.co/index.php/sosquua 


\section{Resumen:}

Este artículo reflexiona acerca de la importancia de los ingredientes de la cocina colombiana como patrimonio cultural. Además, sugiere que el reconocimiento de los mismos debe ser guiado por medio del turismo gastronómico en el país; así, evitando que su uso sea olvidado para las nuevas generaciones, quienes han perdido parte de su identidad nacional al no conocer las raíces de sus ingredientes. Previo a ello, se realizó un análisis de fuentes secundarias que evidencian la importancia de cada uno de los ingredientes en la gastronomía única de las regiones del país; así como la manera en la que se pueden involucrar en el turismo gastronómico.

Palabras clave: ingredientes; patrimonio; gastronomía; turismo.

\section{Abstract:}

This article provides a reflection on the importance of the ingredients of Colombian cuisine as cultural heritage. It also suggests that the recognition of these ingredients should be guided by gastronomic tourism in the country, thus avoiding that their use is forgotten by the new generations, who have lost part of their national identity by ignoring the roots of their ingredients. Previously, an analysis of secondary sources was carried out; it evidences the importance of each one of the ingredients in the unique gastronomy of the regions of the country and the way in which they can be involved in the gastronomic tourism.

Keywords: ingredients; heritage; gastronomy; tourism. 


\section{Introducción}

La importancia de la gastronomía colombiana radica en el uso de sus ingredientes, técnicas, utensilios y recetas que han perdurado en la historia generación tras generación. Por medio de estos, se han desarrollado platos mundialmente reconocidos y apreciados por nacionales e internacionales. Por ejemplo, lo ha sido la bandeja paisa y el ajiaco; especialmente, cuando existe el intercambio cultural promovido por el turismo.

No obstante, es notable el desinterés que las nuevas generaciones tienen respecto al uso de los ingredientes que nacen en Colombia; así como el aprovechamiento y uso de los mismos para la preparación de los platos tradicionales. En este sentido, es posible que se origine por las influencias extranjeras, procesos de la globalización y tendencias, cuyos cambios han tenido grandes afectaciones en la identidad de los jóvenes.

Por tanto, esta investigación se enmarca en el rescate de la importancia de los ingredientes colombianos dentro del marco de un turismo gastronómico accesible a todo público. Principalmente, hacia aquellos que no tienen un conocimiento amplio acerca de su cocina nacional, como es el caso de la descendencia actual. Asimismo, también está dirigido a quienes emprendan una búsqueda donde puedan tener la oportunidad de aprender para recuperar su identidad nacional.

Este es un estudio de tipo reflexivo, se soporta en fuentes secundarias, principalmente artículos, libros, trabajos de grado e información disponible en la web. Se tomó en consideración el aporte de los ingredientes patrimoniales presentes en los platos nacionales y su contribución a la gastronomía nacional. Por medio de las investigaciones, se podrá comprender de manera clara la importancia de resaltar que la memoria debe perdurar en el uso de los ingredientes nacionales y sus tradiciones con los mismos en las actuales y próximas generaciones.

\section{El patrimonio: relación con los ingredientes y la gastronomía tradicional colombiana}

Conocer el patrimonio implica que ha de comprenderse, conservarse y, sobre todo, ser transmitido (Trigueros, 2016). El patrimonio en sí es un bien de valor, que a su vez se hereda y se disfruta. A lo largo de los siglos este concepto ha evolucionado al mismo tiempo que la sociedad. Sin embargo, siempre prevalece la idea de cuidar y proteger nuestro patrimonio, cuyo legado es la herencia de nuestros antecesores (Rico y Baños, 2014; Gutiérrez-Quecano, 2020). 
Asimismo, se pueden encontrar otras innumerables definiciones para describir el patrimonio. Por ejemplo, la Organización de las Naciones Unidas para la Educación y la Cultura (Unesco) describe distintos tipos de patrimonio: patrimonio cultural, patrimonio natural y bienes mixtos. Conviene señalar que el patrimonio cultural inmaterial está conformado por aquellas prácticas culturales, expresiones, saberes o técnicas que son transmitidas de generación en generación. La gastronomía conforma una parte de este conjunto de tradiciones practicadas en nuestro país y en todo el mundo (Inga y Moreno, 2020).

La gastronomía tradicional son los saberes y reflejo de la identidad e historia de los pueblos; se ve manifestado en la preparación de platos tradicionales elaborados con ingredientes de la zona (Robles, 2020). La gastronomía refleja una gran parte de las tradiciones de un pueblo. Aquí se une la riqueza del suelo, los procesos de siembra y recolección de los insumos, el uso de los ingredientes; también las técnicas y saberes que hombres y mujeres han desarrollado durante siglos en el territorio nacional. La gastronomía es identidad, transporta toda la esencia de la cultura del pueblo y posibilita percibir de qué manera viven los habitantes de cada región.

Los platillos típicos de un determinado grupo social esconden años de tradición, recuerdos, momentos, personajes, técnicas y ambientes que hacen que cada uno sea singular y especial. Las fibras de cada plato cuentan el relato sobre cómo dicha comunidad de personas ha sobrellevado una serie episodios que les han permitido llegar a lo que es hoy en día (Valcárcel y Venegas, 2015).

La identidad gastronómica está conectada directamente con los ingredientes que nacen en el país. Por tanto, las prácticas y usos con los mismos generan un sentimiento de pertenencia y se convierten en un símbolo para la región. Así, estableciendo parámetros de preparación (o pasos a seguir) para la construcción de los platos típicos.

La comida colombiana se caracteriza por ser mestiza. Así como el país presenta diferentes factores étnicos, no existe una sola gastronomía, sino diversas gastronomías regionales. Cada una de las regiones posee sus especialidades, en las cuales se mezclan ingredientes indígenas, españoles, con formas de preparación africanas, árabes y españolas; han sido un legado que se conserva hasta hoy (Bogotá Turismo, 2016).

Es cierto que las influencias coloniales brindan una variedad grandísima de tipos de preparación y modos de cocción. Sin embargo, el suelo colombiano es quien brinda la inmensa variedad de ingredientes como frutas, vegetales, hortalizas y especias que están presentes en el diario vivir del colombiano. Su calidad es más que óptima y les hace merecedores de una de las mejores cocinas de Latinoamérica.

Según algunas de las normas técnicas colombianas del Instituto Colombiano de Normas Técnicas y Certificación, un ingrediente es una sustancia o elemento que se emplea en la fabricación o preparación de un alimento. 
Está presente en el producto final, posiblemente de manera modificada o como adición al procedimiento. Al hablar de ingredientes patrimoniales, se hace referencia a esos ingredientes que han hecho parte de la tradición culinaria de las comunidades. Un ejemplo puede ser, en sus múltiples variedades: el maíz, tubérculos como la arracacha, la yuca, el ñame, los cubios; los fríjoles, las lentejas, el cilantro, el romero, el tomillo, el laurel, la canela y los clavos.

La cocina tradicional colombiana, con el uso de los ingredientes patrimoniales, desarrollan prácticas culturales cuyas características resultan de un proceso histórico y común transmitido directamente en la familia; como bien se ha mencionado, pasando de generación en generación. Además, tienen una relación con el ambiente natural donde habita la comunidad, es decir, cada suelo en las regiones es especial para los cultivos de sus propios ingredientes.

Aunque existan similitudes físicas, como es el caso de la producción de plátano en el Urabá antioqueño y el plátano en los Llanos Orientales, o las piñas Perolera y Golden, cultivadas en Santander, Risaralda y Valle del Cauca. El suelo de cada una es el que confiere características en los mismos que permite diferenciar sabores, aromas y texturas particulares en cada ingrediente.

Por consiguiente, desde su crianza todo colombiano debe saber la importancia de la gastronomía nacional; los saberes gastronómicos deben ser parte de la memoria de las nuevas generaciones. Un ejemplo de un aporte a la memoria gastronómica se relaciona con la tradición de las abuelas recreando sus recetas en libros de cocina familiares y posteriormente compartiendo sus saberes a sus hijos y nietos. Entonces, la familia es primordial para el impulso de la memoria culinaria.

Sin embargo, con los fenómenos de la globalización y el deseo del descubrimiento de nuevas culturas, adaptación a nuevas costumbres y tendencias; se ha suprimido la continua identificación, búsqueda y reconocimiento de las raíces propias. Desde años anteriores ha dejado de existir una estrecha relación por la cocina porque se ha perdido el interés de los jóvenes, incluso, de sus padres para comunicar y enseñar sus tradiciones (o lo que estas eran).

Adicionalmente, con la llegada de ingredientes y platos de origen extranjero se ha desplazado el uso de los insumos nacionales y, por ende, el consumo de los platos típicos. Es así como preservar la memoria se convierte en uno de los retos más grandes hoy en día; la importancia de recordar no radica solamente en el saber, también implica valorar lo propio: el suelo, los ingredientes y los platos. De esta manera, con sentido de pertenencia por la gastronomía local hasta llegar al reconocimiento y, entonces, dejar de ser solamente una alimentación básica del día a día y se convertirá en un arte e identidad nacional reconocido por todos. 
Aunque Colombia es un país megadiverso en ingredientes y tradiciones culinarias; al ser olvidados y posiblemente desconocidos para las nuevas generaciones, no hay un sentido de pertenencia profundo con sus insumos y sus raíces. Por ello, recordar la importancia de estos ingredientes debe ser una dirección que el turismo gastronómico puede tener y aprovechar para crecer en el país.

Por lo tanto, como informan Naranjo, Naranjo y Leones (2018), teniendo en cuenta el auge que se está viviendo en torno al turismo gastronómico; se ha visto a la gastronomía como un producto turístico que debe ser explorado al máximo. Colombia tiene todo el potencial para convertirse en uno de los destinos del turismo gastronómico más importantes del mundo.

\section{Turismo gastronómico en Colombia}

De acuerdo con la Organización Mundial del Trabajo (OMT, citado en Mora y Motato, 2019) "el turismo es un fenómeno social, cultural y económico relacionado con el movimiento de las personas a lugares que se encuentran fuera de su lugar de residencia habitual por motivos personales o de negocios/profesionales" (p. 27). De esta forma, el turismo es la vía para posibilitar la relación entre las sociedades y sus diferentes estilos de vida y preferencias.

El turismo en sí, como objeto de estudio, propone una serie de paradigmas, los cuales están orientados desde la inclinación del autor. Es importante concluir que esta actividad en la actualidad ha adquirido una gran importancia, tanto para la economía, como para la sociedad. No solo desde su estudio y conceptualización, sino también desde los turistas, quienes buscan diferentes formas de salir de la rutina y participar activamente en esta actividad (Mora y Motato, 2019, p 27).

La investigación de Oliveira (2011, citado en Torres Bernier, 2003) señala que hay turistas que "se alimentan" y otros que "viajan para comer". Estos turistas, interesados por la comida, realizan su búsqueda desde una perspectiva que puede ser tanto cultural como fisiológica, aunque el objetivo final es encontrar placer. El último caso difiere de los turistas que solo se alimentan, porque estos ven la comida desde una perspectiva fisiológica que no los motiva a viajar.

Sin duda, los turistas gastronómicos son aquellos que buscan comida. En este caso, la gastronomía es el principal atractivo o, usando la terminología que Oliveira (2011) cita de Baptista (1990), el recurso primario del destino visitado. La forma como el turista encara la gastronomía y la intensidad con que ésta afecta su viaje es decisiva para entender el turismo gastronómico.

El turismo gastronómico debe formar parte integrante de la vida local, aporta a la historia, cultura, economía y desarrollo de la sociedad. Adicionalmente, la experiencia del visitante se enriquece por este medio y se establecen relaciones directas con la región, su gente, su cultura y su patrimonio. 
El turismo gastronómico permite un acercamiento a la cultura de un país desde uno de sus aspectos más importantes como lo es su cocina. Adicionalmente, permite a los mismos pobladores del país indagar más acerca de su historia culinaria.

Así como lo expresa Llano (2017), Colombia presenta una gran variedad culinaria gracias a sus ventajas agrícolas, ganaderas, pesqueras, históricas, socioculturales, entre otras. Por ello, difundir la gastronomía colombiana por medio de la actividad turística permite el reconocimiento de los patrimonios gastronómicos locales. En el marco de unas potencialidades geográficas y culturales, pueden publicitar un lugar como fuente de una experiencia turística auténtica y memorable.

Entonces, al hablar de un desarrollo turístico enfocado en la gastronomía, como parte de un proceso de crecimiento en el país; el patrimonio gastronómico puede contribuir no solo al incremento de la economía local. También al complemento de las actividades turísticas que se han venido desarrollando con el aprovechamiento de los destinos nacionales.

Con el desconocimiento de los ingredientes nacionales, el turismo gastronómico tiene una nueva oportunidad de permitirle al público acercarse a sus raíces. Con actividades dirigidas a indagar el origen, identificación y uso de los ingredientes que forman parte del patrimonio cultural, se podrán crear estrategias para incentivar el consumo de los platos típicos y el uso de sus ingredientes en el diario vivir del habitante colombiano y, finalmente, continuar creando un acercamiento a la cultura.

Un ejemplo de ello ha sido el proyecto que ha realizado la dirección de patrimonio del Ministerio de Cultura en el año 2013 en su Política para el conocimiento, salvaguardia y fomento de la alimentación y las cocinas tradicionales en Colombia. Aunque este no tiene fines turísticos, se adentra a brindar una explicación clara y fomentar estrategias para el reconocimiento de las cocinas tradicionales en el país, desde las bases políticas, históricas y culturales.

El turismo gastronómico tiene un papel importante en la valorización y promoción de la gastronomía. Es así como esta actividad puede convertirse en un promotor del desarrollo de la cultura y la percepción de la misma crece de manera positiva en lugares cuya riqueza gastronómica es increíble. Adicionalmente, contribuye a la economía de los habitantes del destino.

Un ejemplo de turismo gastronómico en Colombia son las rutas del café en el eje cafetero. Allí se muestra el proceso de siembra del café, hasta la degustación de una aromática taza de su bebida. Sin embargo, Colombia cuenta con un sinnúmero de ingredientes que hacen parte de la vida local de muchos pueblos. Tal es el caso de la Panela, el Sagú, el queso Paipa, el bocadillo, hibias y chuguas, entre otros; son la base para crear deliciosos platos y productos autóctonos de las regiones nacionales. 
Por otra parte, al encontrar destinos menos reconocidos pero ricos en cultura y tradiciones gastronómicas, como es el caso del municipio de Fomeque, en Cundinamarca, con sus productos a base de Sagú (pan de Sagú). En este sentido, las tradiciones se mantienen y sus habitantes tienen un sentido de pertenencia por sus ingredientes y productos.

Así, se encuentra la oportunidad de crear vías de promoción y desarrollo para darlos a conocer y realzar su valor cultural, por medio de ferias y eventos gastronómicos, o rutas turísticas donde el origen de un producto sea el protagonista principal. De la misma manera, el interés del visitante por las comidas puede fomentar la participación de las comunidades, quienes sentirán que son importantes al igual que sus tradiciones y serán motivados para desarrollar y continuar prácticas dirigidas al turismo gastronómico.

La planeación y creación de nuevos proyectos que vinculen al turismo y la gastronomía liderados por los jóvenes estudiantes, profesionales en turismo, cocineros, investigadores y las comunidades, permitirá que se preserve nuestro patrimonio inmaterial con la cocina tradicional. Asimismo, continuar brindando momentos inolvidables en la experiencia del visitante para detener el desconocimiento de los ingredientes propios en las nuevas generaciones.

El turismo valoriza lo original, único y diferente de las comunidades. El turista es quien se deja guiar a través de los sencillos platos y sus ingredientes típicos. Con agrado se da la oportunidad de conocer lo que los nacionales rechazan por ser común para ellos y se resisten a darle la importancia debida.

La motivación de viajar en estos días aumenta en los jóvenes. Ellos quieren vivir experiencias enriquecedoras para su vida. Sin embargo, desconocen su historia y por ende sus ingredientes. Lo anterior, teniendo en cuenta que la alimentación será un servicio que el sistema turístico prestará sin lugar a dudas. Entonces, el turismo gastronómico puede involucrarse y hacer buen uso de las nuevas tecnologías para transmitir conocimiento y fomentar el valor que tiene la cocina nacional.

\section{Conclusiones}

El turismo gastronómico en Colombia es una actividad que permite acercarse a la historia de un pueblo desde sus raíces gastronómicas e ingredientes propios. Además, es una actividad que aporta a la economía de la región y el país.

Al notarse una pérdida de la identidad nacional, se crea la oportunidad de incentivar nuevas estrategias para contribuir al reconocimiento de los ingredientes. De esta manera, influencia a las nuevas generaciones del país, por medio de las nuevas tecnologías, para entender su patrimonio cultural y continuar promoviéndolo. 
Aunque existen proyectos referentes a la cocina tradicional, hace falta la creación de más programas dirigidos al turismo gastronómico en destinos menos conocidos. Es así como podría surgir la oportunidad de jóvenes estudiantes y profesionales en turismo de trabajar junto con las comunidades para realzar sus ingredientes y platos típicos. Con estas acciones, Colombia podrá ser reconocido como un país que conoce la historia de su cocina e invierte en la conservación de su patrimonio continuamente a través de los años.

\section{Referencias bibliográficas}

Bogotá Turismo. (2016). Turismo gastronómico. Bogotá Turismo. Recuperado de http://www.bogotaturismo.gov.co/Turismo\%20Gastron\%C3\%B3mico

Gutiérrez-Queccano, R. (Comp.). (2020). El turismo gastronómico sostenible. Ruta Bochica - Potencialidades y modelo pedagógico para su desarrollo en Colombia. Bogotá: Editorial Fundación Universitaria San Mateo. Recuperado de https://palma.sanmateo.edu.co/

Llano, F. (2017). Gastronomía, turismo y potencialidades territoriales: el plato minero y la salazón, bases para el turismo alimentario en Nemocón. Cuadernos de Geografía: Revista Colombiana de Geografía, 26(2), pp. 295-306.

Mora, J.A. y Motato, J. W. (2019). Turismo comunitario en Colombia desarroIlo social y sostenibilidad. Bogotá: Corporación Universitaria Minuto de Dios.

Inga, C. y Moreno, P. (2020). Patrimonio cultural inmaterial y turismo gastronómico de la parroquia Yaruquíes del cantón Riobamba (trabajo de grado). Universidad Nacional de Chimborazo, Riobamba, Ecuador.

Naranjo, R., Naranjo, G., y Leones, A. (2018). La gastronomía colombiana. Atractivo turístico en crecimiento. Hitos de Ciencias Económico Administrativas, 24(68). Recuperado de: http://revistas.ujat.mx/index.php/hitos/ article/view/2509/1921

Oliveira, S. (2011). La gastronomía como atractivo turístico primario de un destino: El Turismo Gastronómico en Mealhada-Portugal. Estudios y 
perspectivas en turismo, 20(3), pp. 738-752.

OMT. (2019). Guía para el desarrollo del turismo gastronómico. Madrid: OMT y BCC. Recuperado de https://www.e-unwto.org/doi/ $\mathrm{pdf} / 10.18111 / 9789284420995$

Rico, E. y Baños, C. (2014). El patrimonio cultural en los procesos de renovación de áreas turísticas litorales. Una aproximación al destino turístico de la Costa Blanca (Alicante, España). Cuadernos Geográficos 55(2), pp. 299-319.

Robles, O. (2020). La gastronomía tradicional como patrimonio cultural inmaterial y factor de desarrollo turístico de la comunidad de Agato-Otavalo (trabajo de grado). Universidad Técnica del Norte, Ibarra, Ecuador. Recuperado de http://repositorio.utn.edu.ec/handle/123456789/10492

Torres Bernier, E. (2003). Del turista que se alimenta al turista que busca comida - Reflexiones sobre las relaciones entre la gastronomía y el turismo. En G. Lacanau y J. Norrild (comp.), Gastronomía y turismo: cultura al plato (pp. 305-320). Buenos Aires: CIET.

Trigueros, C. (2016). La arqueología como factor de desarrollo del turismo local. International Journal of Scientific Management Tourism, 2(3), pp. 355-368.

Valcárcel, D. y Venegas, A. (2015). La comida típica dentro de la internacionalización de la oferta gastronómica en Bogotá. Turismo y Sociedad, 16, pp. 187-198. DOI: http://dx.doi.org/10.18601/01207555.n16.10 